\title{
Beam steering and monopulse processing of probe-fed dielectric resonator antennas
}

\author{
S.P. Kingsley and S.G.O'Keefe
}

\begin{abstract}
It is shown that the azimuth radiation pattern direction of a multi-probe dielectric resonator antenna (DRA) can be changed by exciting different probes within the dielectric when the other probes are open-circuited. When two probes are excited simultaneously, smaller steering increments can be obtained without significantly changing the beamwidths. In some combinations, a pair of probes has a significantly greater bandwidth than a single probe. Driving pairs of probes with unequal power division is shown to steer the beam by the expected amount, thus forming the basis for future electronic beam steering of DRAs and creating the possibility of obtaining any desired steering angle. DRA monopulse processing, using two beams to form sum and difference patterns, is also demonstrated. These results have obvious applications for radar systems where control of the radiation pattern is desirable. The efficiency of DRAs make them well suited to these applications, except where large bandwidths are required.
\end{abstract}

\section{Introduction}

Since the first systematic study of dielectric resonator antennas (DRAs) [1]; interest has grown in their radiation patterns because of their high radiation efficiency, their good match to most commonly used transmission lines and their small physical size [2]. Most reported configurations have used a slab of dielectric material mounted on a ground plane, excited by either a slot antenna in the ground plane or by a probe inserted into the dielectric material. A few experiments have been reported using two probes fed simultaneously in a circular dielectric slab. These probes were installed on radials at $90^{\circ}$ to each other and fed in anti-phase to create circular polarisation $[3,4]$, and one publication included the concept of switching probes on and off [5]. In this paper, we report on a DRA with several probes fed in such a way that the antenna pattern can be steered. We also discuss the use of two probes driven simultaneously in-phase and $180^{\circ}$ out of phase in order to generate monopulse sum and difference patterns.

A simple method of electronically steering an antenna pattern is to have a number of existing beams and to switch between them, or to combine them to achieve the desired beam shape. A circular DRA fed by a single probe towards the edge of the dielectric, and tuned to excite the fundamental $\mathrm{HEM}_{11 \delta}$ mode, produces a vertically polarised radiation pattern which has a figure-of-eight shape in the azimuth [1]. Modelling of cylindrical DRAs by FDTD (finite difference time domain), to be reported elsewhere, shows that if several such probes are inserted into the dielectric and one is driven while all the others are open-

(C) IEE,1999

IEE Proceedings online no. 19990307

DOI: 10.1049 /ip-rsn:19990307

Paper first received 30th June 1998 and in revised form 12th February 1999

S. P. Kingsley is with the Department of Electronic and Electrical Engincering, University of Sheffield, Mappin Street, Sheffield S1 3JD, UK.

S. G. O'Keefe is with the School of Microelectronic Engineering, Griffith University, Nathan QLD 4111, Australia. circuit, then the beam direction can be moved by switching different probes in and out. To investigate whether this result could be implemented in practice, and to find out if two probes could be driven simultaneously with different powers to produce a combined pattern in any given direction, the idea was tested using a VHF DRA on the Sheffield University outdoor antenna range at Harpur Hill, Buxton.

\section{Measured radiation patterns}

One simple way to experiment with DRAs is to use distilled water as the dielectric medium. The use of a liquid dielectric has many advantages in the initial stages of a DRA design; the dielectric can easily be constrained to the desired shape; there are no airgaps between the probe and the dielectric, or between the dielectric and the conducting plane; when dealing with physically large DRAs at low frequencies, distilled water is a low cost and readily accessible material with a high dielectric constant.

Fig. 1 shows the structure of the water-filled test antenna. It is excited by a coaxial feed connected to one

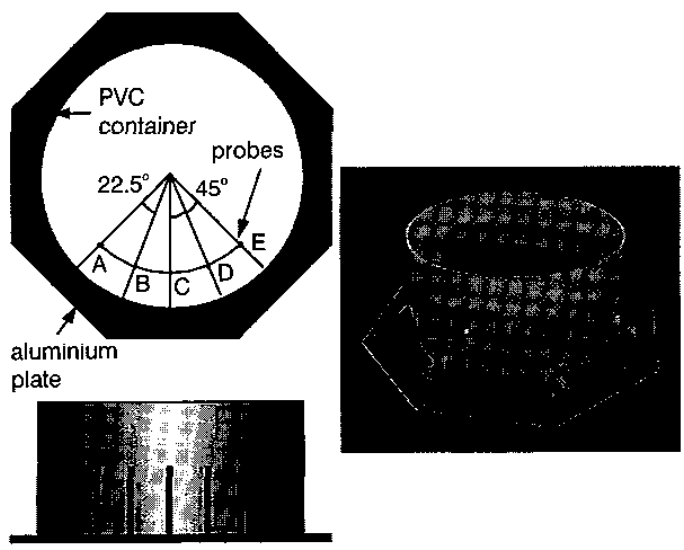

Fig. 1 Structure of water-filled multi-probe cylindrical VHF DRA 


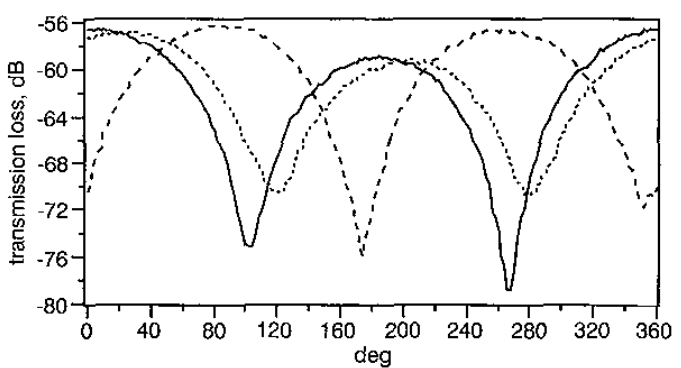

Fig. 2 Measured azimuth radiation patterns for probe positions $C, D$ and $E$

The vertical scale is the transmission loss given by the HP8714 spectrum analyser and represents the total loss between the terminals of the instrument.

$-\mathrm{C}$

… D

$--\mathrm{E}$

of five probes located near the outer edge of the dielectric. The water dielectric is contained in a $550 \mathrm{~mm}$ diameter PVC-walled container, of thickness $5 \mathrm{~mm}$, mounted on an octagonal aluminium plate of dimensions $800 \mathrm{~mm}$ across the flats. The container was filled to a depth of $200 \mathrm{~mm}$. The relative permittivity of the water used in the experiments, at a temperature of $15^{\circ} \mathrm{C}$ and at a frequency of between 10 and $100 \mathrm{MHz}$. was calculated to be 84 . The conductivity of the water was about $10 \mu \mathrm{S}$. The unloaded Q factor of the created DRA was approximately 28.4.

The impedance of the DRA was measured using a HP8714 network analyser, with the probe length adjusted to achieve the best return loss. For probes $\mathrm{C}, \mathrm{D}$ and $\mathrm{E}$ in Fig. 1 the return losses were $-26 \mathrm{~dB},-32 \mathrm{~dB}$ and $-13 \mathrm{~dB}$, respectively. The corresponding resonant frequencies were $55.24 \mathrm{MHz}, \quad 55.27 \mathrm{MHz}$ and $56.59 \mathrm{MHz}$. The two imbedded probes $\mathrm{C}$ and $\mathrm{D}$ therefore behaved in a similar way, but the end probe $\mathrm{E}$ was significantly different, as might perhaps be expected. The end probe effect is unlikely to occur in an operational system, where an entire ring of probes might be used such that each one is equally imbedded.

An outdoor test range was used to measure the radiation patterns for each probe position as a function of azimuth at a fixed elevation angle of $2^{\circ}$ above horizontal. Measurements were taken using a HP8714 network analyser and a test monopole tuned to the resonant frequency. Fig. 2 shows the patterns for the probe positions C, D and E. The expected figure-of-eight pattern, due to the magnetic dipole distribution within the DRA, is evident for these positions. If these probes were present in isolation, they would create beams with bore-sights on $0^{\circ}, 22.5^{\circ}$ and $45^{\circ}$, respectively, but this is clearly not the case. A single probe located at position $\mathrm{C}$ would give maxima at $0^{\circ}$ and $180^{\circ}$ and nulls at $90^{\circ}$ and $270^{\circ}$ : when measurements are made with the other probes present, but open-circuited, they are quite close to these values. The pattern for probe $\mathrm{D}$ shows a somewhat larger variation from the isolated probe case, but errors are less than $8^{\circ}$. Probe E, however, has a radiation pattern steered $85^{\circ}$ instead of the isolated probe value of $45^{\circ}$, and there is clearly an end effect perturbing the pattern of this probe. Probe A at $-45^{\circ}$ (not plotted in Fig. 2) showed exactly the same effect with a pattern that is the mirror image of that for probe $\mathrm{E}$.

\section{Exciting two probes simultaneously}

It has been demonstrated that the antenna radiation pattern can be steered incrementally by selecting the appropriate probe. The next step was to see if more than one probe could be excited simultaneously to produce a pattern with a smaller steering increment. If probes $B$ and $D$ are driven simultaneously, the resulting pattern should have a boresight along $0^{\circ}$, which can be compared with the pattern for probe $\mathrm{C}$ alone; the same is true of patterns $\mathrm{A}$ and $\mathrm{E}$. To test this concept, the test range was set up with a power splitter-combiner to divide the signal from the network analyser between two probes. The splitter-combiner exhibits only a small insertion loss and, at $60 \mathrm{MHz}$, a roughly equal phase shift of about $3^{\circ}$ between the common port and the two probe ports. The measurements taken with this arrangement for probes $\mathrm{B}+\mathrm{D}$ and $\mathrm{A}+\mathrm{E}$ are shown in Fig. 3 , together with the pattern of probe $C$ for comparison. The pattern for $C$ has $a-3 \mathrm{~dB}$ beamwidth of $92^{\circ}$, whereas $\mathrm{B}+\mathrm{D}$ has $92^{\circ}$ and $\mathrm{A}+\mathrm{E}$ has $88^{\circ}$.

The return losses for probes $\mathrm{C}, \mathrm{B}+\mathrm{D}$ and $\mathrm{A}+\mathrm{E}$ were also measured and are shown in Fig. 4. Probe $C$ has a bandwidth of $944 \mathrm{kHz}$ and B $+\mathrm{D}$ has a bandwidth $20 \%$ greater at $1.13 \mathrm{MHz}$, only a small part of which can be accounted for by the slight increase in resonant frequency. The bandwidth of the A+E combination is less at 750 $\mathrm{kHz}$, but this might be expected as the radiation patterns are orthogonal and the probes have little mutual interaction. It is thus concluded that, when two probes are excited simultaneously, the patterns have slightly less gain than for a single probe, have patterns with similar beamwidths, but may have a significantly greater bandwidth if the angle subtended between them is not too great.

Other pairs of probe combinations can be driven. Fig. 6 shows the theoretical patterns for $\mathrm{B}+\mathrm{C}, \mathrm{B}+\mathrm{D}, \mathrm{B}+\mathrm{E}$ and $\mathrm{A}+\mathrm{E}$, which ideally would give lobes centred on $0^{\circ}$, $-11.25^{\circ}, 0^{\circ},+11.25^{\circ}$ and $0^{\circ}$, respectively. These patterns

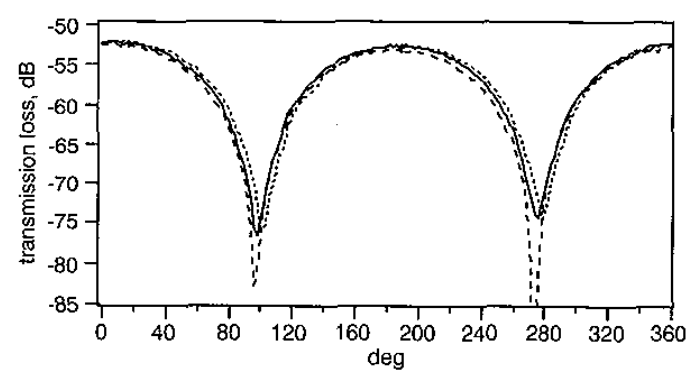

Fig. 3 Measured azimuth radiation patterns for probes $C, B+D$ and $A+E$

$-\mathrm{C}$

$\ldots-\mathrm{B}+\mathrm{D}$

$---\mathrm{A}+\mathrm{E}$

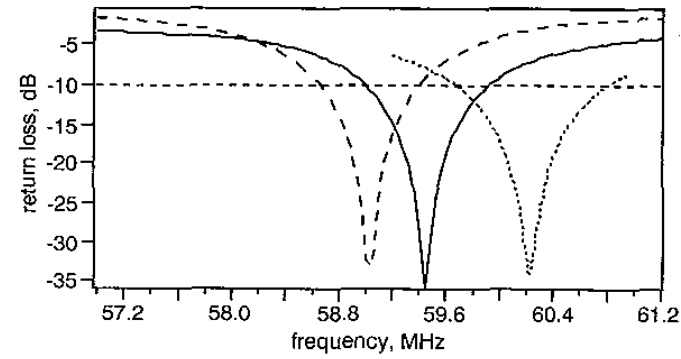

Fig. 4 Measured return loss and bandwidths for probes $C, B+D$ and $A+E$

$-\mathrm{C}$

$-\cdots B+D$

$---\mathrm{A}+\mathrm{E}$ 

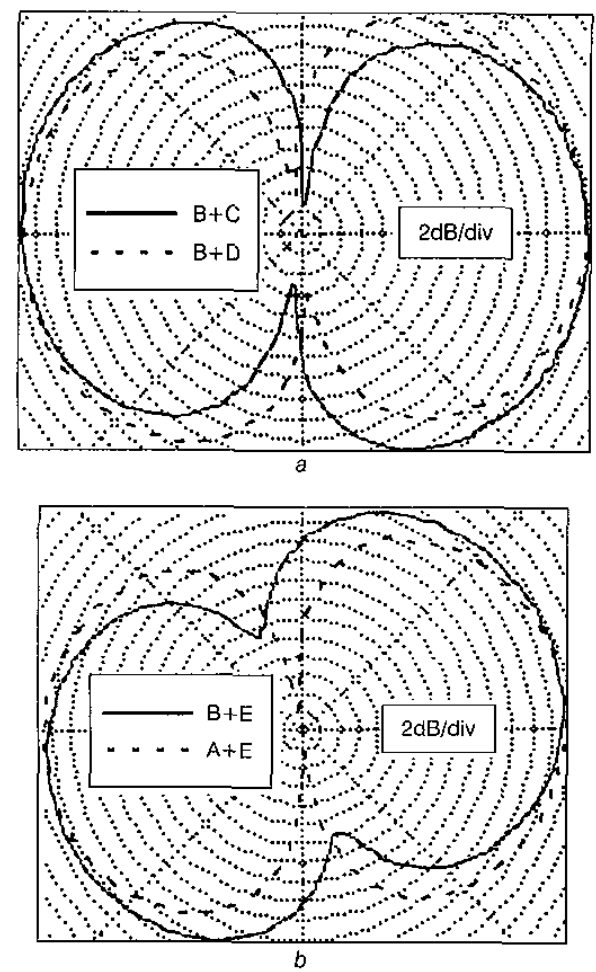

Fig. 5 Measured beam patterns for probes $B+C, B+D, B+E$ and $A+E$
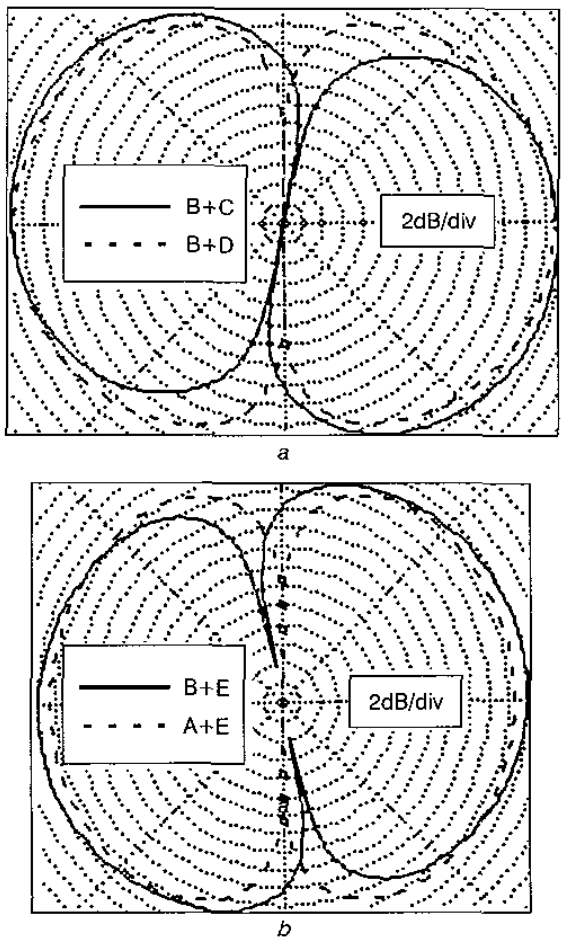

Fig. 6 Expected beam patterns calculated for probes $B+C, B+D$, $B+E$ and $A+E$

were generated by taking an ideal $\operatorname{cosine}(\varphi)$ function with a finite null for one probe position, then rotating it to another and voltage summing (signed but without noise added). The expected lobes and nulls are observed with the nulls of the summed patterns deeper than those of the single probe pattern because the voltage sign changes between the front and the back lobe and some cancellation occurs at the overlap between patterns.

Care must be taken when interpreting the meaning of null depths measured on an outdoor test range because the maximum depth of nulls is limited by noise, background radio traffic and the finite dynamic range of the measurement system. In addition, the automated measurement system samples the radiation pattern only every $2^{\circ}$, and so deep nulls can be missed if they fall between sample points. Nonetheless, fairly deep nulls are sometimes observed.

Fig. 5 shows the measured azimuth patterns corresponding to the calculated patterns, and there is remarkable agreement between Figs. 5 and 6 . The measured null for probes $\mathrm{A}+\mathrm{E}$ is very deep, dropping below the bottom of the Figure to $-95 \mathrm{~dB}$, but the null for probes $\mathrm{B}+\mathrm{E}$ is less deep than expected.

\section{Beam steering by weighted addition}

Some modern in-service radars use a resistive beam forming matrix to combine the signals from different antenna elements, with appropriate weighting, to form the desired radiation pattern. To test this concept using a DRA, a second power splitter-combiner was employed with one port terminated (as shown in insert in Fig. 7) in order to drive probe $\mathrm{E}$ simultaneously with probe $\mathrm{B}$ but at half the power. This arrangement was expected to produce a main lobe at an angle of $+1.3^{\circ}$, but the pattern for $\mathrm{B}+\mathrm{E}$ equally weighted should have a main lobe at $-11.25^{\circ}$. The weighting process should therefore rotate the beam by about $12.5^{\circ}$.

The measured azimuth radiation patterns for $\mathrm{B}+\mathrm{E}$ and $\mathrm{B}+\mathrm{E} / 2$ can be compared in Fig. 7. and it is clear that the beam has moved by at least $10^{\circ}$. Both patterns are slightly shifted to the right; this is believed to be a range effect at this particular frequency. The power lost in the $50 \Omega$ termination (insert in Fig. 7) means the gain of the $\mathrm{B}+\mathrm{E} / 2$ pattern should be about $1.5 \mathrm{~dB}$ less than the $\mathrm{B}+\mathrm{E}$ pattern. In fact, the loss is somewhat greater than this, at nearly $2 \mathrm{~dB}$, because of the insertion loss of the power splitter-combiners.

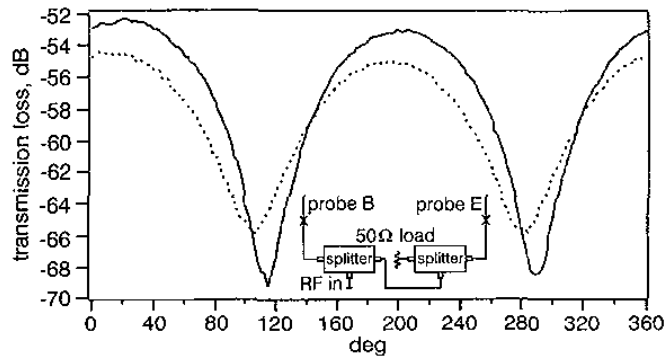

Fig. 7 Measured azimuth radiation patterns for probes $B+E$ and $B+E / 2$

The inscrt shows the arrangement used to halve the power to probe $E$ $-\mathrm{B}+\mathrm{E}$ -.. $\mathrm{B}+\mathrm{E} / 2$

\section{Monopulse processing}

Monopulse processing, perhaps better described as simultaneous lobing, is a technique extensively used in the radar 
field for target tracking [6] and has been known since 1928 [7]. Two primary antenna patterns, offset in angle, are generated simultaneously (either electronically or computationally after A/D conversion) and are added to create a sum channel $\Sigma$ with a good signal-to-noise ratio. This channel is used to detect targets, and measure range and Doppler information. A difference beam $\Delta$ with a sharp null at the centre is used to measure angular information. When a target is in the null at the centre of the pattern, no error voltage is generated. If the target drifts to one side or the other, a plus or minus voltage occurs. In principle, the $\Delta$ beam alone can be used to indicate when the target is on boresight, but it cannot be used to indicate how far a target is off boresight because the echo amplitude depends on many other factors. By using $\Sigma$ to normalise $\Delta$, the offboresight deviation off the target $\theta$ can be reliably obtained, despite echo amplitude variations, from the ratio

$$
\frac{\Delta}{\Sigma}=k 0
$$

where $k$ is the slope of the real part of the voltage $\Delta / \Sigma$ curve near $\theta=0$, when $\theta$ is in $\operatorname{rad}$.

We have not found published examples of monopulse processing with DRAs. There have been descriptions of feeding $90^{\circ}$ probes with orthogonal phases to create circular polarisation in the $\mathrm{HE}_{11 \delta}$ mode $[3,4]$ and the concept of switching probes on and off [5]. However, there appear to be no reports concerning feeding probes in antiphase to create monopulse patterns. FDTD simulations of DRAs showed the theoretical feasibility of this technique, and so an experiment was designed to see if it could be demonstrated in practice using the $60 \mathrm{MHz}$ water-filled antenna and the Buxton test range. For each measurement, two probes were fed using a power splitter-combiner to divide the signal from the network analyser between them. When measuring the difference patterns, an extra half wavelength section of coaxial cable was inserted between the power splitter-combiner and one of the probes.

Figs. 8 and 9 show the measured sum and difference azimuth radiation patterns for probes $\mathrm{B} \pm \mathrm{C}, \mathrm{B} \pm \mathrm{D}, \mathrm{B} \pm \mathrm{E}$ and $A \pm E$, which are separated in the DRA by angles of $22.5^{\circ}, 45^{\circ}, 67.5^{\circ}$ and $90^{\circ}$, respectively. In all four cases, it is clear that monopulse sum and difference patterns are
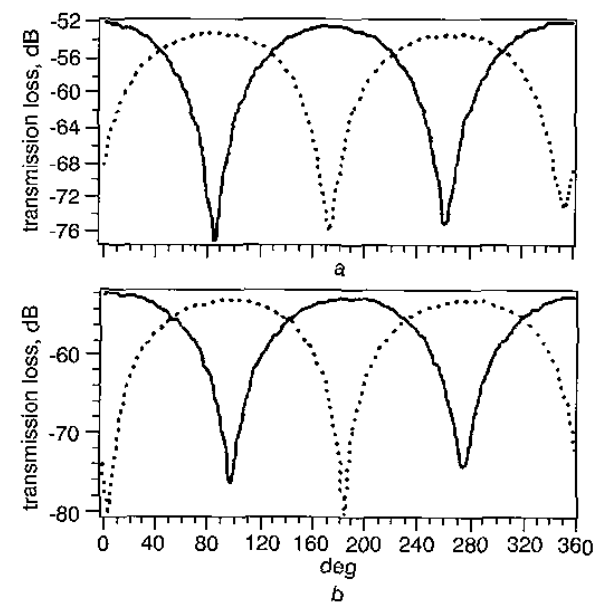

Fig. 8 Measured sum and difference azimuth radiation patterns for probes $B \pm C$ and $B \pm D$

$$
\text { - sum }
$$$$
\text { -..- difference }
$$

$a \mathrm{~B} \pm \mathrm{C}$

$b \mathrm{~B} \pm \mathrm{D}$
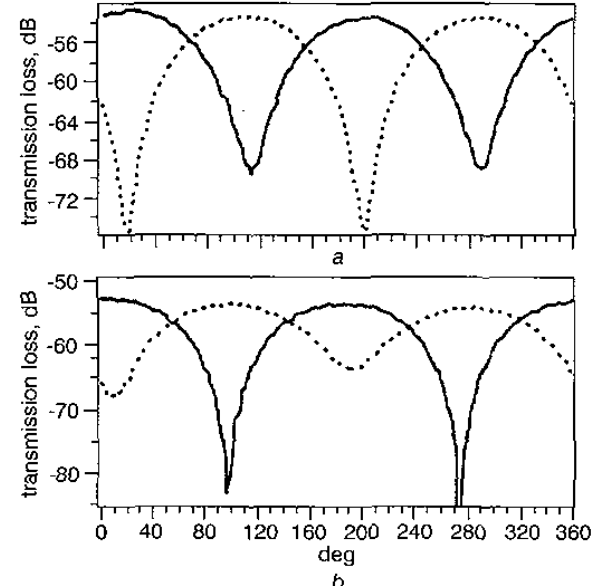

Fig. 9 Measured sum and difference azimuth radiation patterns for probes $B \pm E$ and $A \pm E$

sum

.... difference

$a \mathrm{~B} \pm \mathrm{E}$

$b \mathrm{~A} \pm \mathrm{E}$

created, although the patterns for $\mathrm{A} \pm \mathrm{E}$ (Fig. $9 b$ ) are of little practical use as the primary (single probe) beams are at $90^{\circ}$ to each other. Within the DRA, the probes are electrically closely spaced, only $0.15 \lambda$ apart. This means that for the adjacent probes $\mathrm{B}$ and $\mathrm{C}$ there is significant mutual coupling, and the difference pattern null is not as deep as for probes $B$ and $D$, which are twice as far apart and have quite useful monopulse sum and difference radiation patterns. This result is consistent with good monopulse design practice of offsetting the two primary beams by about half a beamwidth.

\section{Monopulse accuracy}

Figure-of-eight DRA patterns with main lobes $90^{\circ}$ wide are clearly not ideal for direction finding, and even the created monopulse nulls are much less sharp than would be used in an operational microwave tracking radar. The advantage of the demonstrated DRA monopulse system, however, is that it can be extended to lower frequencies such as the HF band, where it is very difficult to generate these patterns unless a lot of space is available to erect a large antenna (small crossed loop antennas can be used, but they are unacceptably lossy for transmission).

The limitation to, the accuracy of a radar system is the Cramer-Rao (C-R) bound [8]. Strictly, the C-R bound applies to high signal-to-noise cases where there is only one unknown parameter being estimated in the presence of additive white Gaussian noise. This last condition is rarely fulfilled at low frequencies where radio systems tend to be externally noise-limited, but these more complicated situations lead to higher errors and the C-R bound can still be taken as representing the lowest obtainable uncertainty. A simple way of expressing the $\mathrm{C}-\mathrm{R}$ bound is to say that, if an unknown parameter $M$ is estimated with a basic resolution step size of $\Delta M$, then the lower bound to the RMS uncertainty in the estimate $\delta M$ is given by

$$
\delta M \approx \frac{\Delta M}{\sqrt{2 . S N R}}
$$

where SNR is the signal-to-noise ratio (linear, not in $\mathrm{dB}$ ). In the monopulse case, where information from two 
antenna beams is combined, the angle RMS error $\delta \theta$ is given by

$$
\delta 0 \approx \frac{\Delta \theta}{k \sqrt{2 \cdot S N R}}
$$

where $\Delta \theta$ is the $3 \mathrm{~dB}$ beamwidth and $k$ is the slope as defined in eqn. 1 [9] or Section 18.10 in [10] for more details. For the DRA B-D pattern, $k$ was found to be approximately 0.97 , as measured at the centre of a linear voltage plot. For a SNR of $20 \mathrm{~dB}$, the lower error bound can be calculated from eqn. 3 to be about $6.6^{\circ}$. In this example, the offset angle between beams is $45^{\circ}$ (roughly half a beamwidth); a wider offset angle would give a larger value of $k$ and reduce the error, but at the same time the crossover loss would increase and reduce the SNR, thereby increasing the error.

A $6.6^{\circ}$ error is far too large for a conventional tracking radar. However, it may well be useful for HF radar, where the focus is usually on the detection and tracking of targets below the microwave horizon, rather than engaging in high-accuracy close-range tracking.

\section{Conclusions}

Measurements made on an outdoor test range confirm FDTD simulation results; a dielectric resonator antenna can be fitted with several probes simultaneously so that the azimuth radiation pattern main lobe direction can be changed by exciting a different single probe with the other probes open-circuited. The measurements show that the end probe of the array behaves in a different way to imbedded probes and that the resonant frequency varies slightly with the probe position. This is not expected to be a problem in an operational system, where there would be a complete ring of probes iteratively tuned to the same frequency and all equally imbedded.

Two probes can be excited simultaneously to obtain smaller steering increments without significantly changing the beamwidths. Very good agreement has been shown between the expected patterns and those actually measured on an outdoor test range. In some combinations, a pair of probes has been shown to produce a significantly greater bandwidth than a single probe.

Driving pairs of probes with unequal power division has been shown to steer the beam by the expected amount, thus forming the basis for future weighted beam forming with
DRAs and the possibility of obtaining any desired steering angle.

Monopulse processing, a technique used extensively by tracking radars to determine the angular position of targets, has been shown to be practicable for a VHF DRA during field trials. This has the important implication that the technique can be extended to lower frequencies. Angular uncertainties in locating a radio source with such a system should be better than $10^{\circ}$ and could be as low as a few degrees under good signal-to-noise conditions when a single source is present.

All these results combine to show that there is no obstacle to electronically steering the beam of a DRA azimuth radiation pattern or creating a null at will. This has obvious applications for radar and communication systems, where it may be desirable to transmit, or avoid transmitting, in certain directions. The efficiency of DRAs make them well suited to these applications, except where large bandwidths are required. If the DRA is used in receive-only mode, then the output of the probes can be recorded by separate receivers and the beams formed electronically either in real-time or post-processed, as required.

\section{References}

1 LONG, S.A., McALLISTER, M.W., and SHEN, L.C.: 'The resonant cylindrical dielectric cavity antenna', IEEE Trans. Antennas Propag., 1983, AP-31, pp. 406-412

2 MONGIA, R.K., and BHARTIA, P. 'Dielectric resonator antennas -a review and general design relations for resonant frequency and bandwidth', Int. J. Microw. Millim-Wave Comput.-Aid. Eng., 1994, 4, (3), pp. width', Int

3 MONGIA, R.K., ITTIPIBOON, A., CUHACI, M., and ROSCOE, D. 'Circular polarised dielectric resonator antenna', Electron. Lett., 1994 , 30, (17), pp. 1361-1362

4 DROSSOS, G., WU, Z., and DAVIS, L.E.: 'Circular polarised cylindrical dielectric resonator antenna', Electron. Lett., 1996, 32, (4), pp. 281283

$S$ DROSSOS, G., WU, Z., and DAVIS, L.E.: 'Switchable cylindrical dielectric resonator antenna', Electron. Lett., 1996, 32, (10), pp. 862864

6 KINGSLEY, S.P., and QUEGAN, S.: 'Understanding radar systems' (McGraw-Hill, London, UK, 1992, 1st edn.)

7 RHODES, D.R.: 'Introduction to monopulse' (McGraw-Hill, New York, 1959, 1st edn.)

8 COOK, C.E., and BERNFIELD, M.: 'Radar signals, an introduction to theory and application' (Academic Press, New York, 1967)

9 LEVANON, N.: 'Radar principles' (John Wiley, New York, 1988, 1st edn.)

10 SKOLNIK, M.I.: 'Radar handbook', (McGraw-Hill, New York, 1990, 2nd edn.) 